\title{
Use of a tubular retractor system in microscopic lumbar discectomy: 1 year prospective results in 135 patients
}

\author{
Sylvain Palmer, M.D. \\ Mission Hospital Regional Medical Center, Mission Viejo, California
}

\begin{abstract}
Object. Application of minimally invasive techniques to lumbar disc surgery has led to the development of the Microscopic Endoscopic Tubular Retractor System (METRx-MD). A prospective evaluation of results and complications was undertaken.

Methods. One hundred thirty-five patients underwent surgery in which the METRx-MD system was used; most procedures were performed on an outpatient basis, and general anesthesia was induced in all cases. All patients were followed prospectively. Outcomes were measured using a visual analog scale (VAS), the Oswestry Disability Index (ODI), and the Short Form-36 (SF-36) questionnaires. Follow-up data were collected by an outside company, which also tabulated the data.

Data were collected in 129 of 135 patients. Improvement was seen on the VAS (Scores 7-2), ODI (Scores 57-16), and SF-36 scales (bodily pain Scores 20-60). Patient satisfaction with results was 94\% and with office services $88 \%$. Thirty-six percent of patients returned to work at 0 to 2 weeks, $38 \%$ at 3 to 5 weeks, and $25 \%$ at 5 to 26 weeks. Hospital charges decreased by $\$ 2395(18 \%)$.

The mean operative time was 66 minutes, and the mean blood loss was $22 \mathrm{ml}$. Complications included one superficial wound infection, one discitis, three durotomies, and three cases of excessive bleeding $(>100 \mathrm{ml})$. There were five reoperations: four for recurrent disc herniations, (two ipsilateral and two contralateral to the index site) and one for spinal stenosis contralateral to the index site.

Conclusions. Minimally invasive surgery in which the METRx-MD system is used is clinically effective and cost effective. Patient satisfaction was high. A mean per case cost savings of $\$ 2395$ was realized. Complications rates were comparable with those associated with traditional microdiscectomy procedures.
\end{abstract}

\section{KEY WORDS - lumbar herniated disc - microdiscectomy - minimally invasive surgery • outpatient surgery $\bullet$ operative microscope}

Low-back pain is a serious public health problem resulting in estimated direct and indirect costs of up to $\$ 3$ billion. ${ }^{9}$ It is often the result of lumbar disc disease. ${ }^{13,14}$ The natural history of herniated lumbar discs is one of spontaneous regression. ${ }^{19} \mathrm{~A}$ variety of modalities have been shown to be effective in the conservative management of acute low-back pain. ${ }^{21}$ Weber $^{22}$ has shown that surgery yielded better results than conservative care at 1 year posttreatment but that after 4 years this difference was no longer statistically significant. Nonetheless, 185,000 patients underwent surgical intervention in 1990 in the United States, and the rate escalated $40 \%$ between 1979 and $1990 .^{4}$

In 1934, Mixter and Barr ${ }^{12}$ first reported the surgical treatment of patients with lumbar herniated discs by undertaking laminectomy and discectomy. Caspar ${ }^{2}$ in 1977 and Williams ${ }^{23}$ in 1978 reported refinements in approach

Abbreviations used in this paper: LOS = length of hospital stay; MED = microendoscopic discectomy; METRx-MD = Microscope Endoscopic Tubular Retractor System-Microdiscectomy; ODI = Oswestry Disability Index; SF-36 = Short Form-36; VAS = visual analog scale. with the use of a microsurgical technique. Increasingly, minimally invasive techniques have been applied to spinal surgery, ${ }^{11,16,20}$ many of which have suffered from the inability to allow direct visualization of the pathological entity and the neural elements, thereby limiting their application and effectiveness. In 1997, Foley and Smith ${ }^{8}$ introduced MED. The original MED instrumentation set was modified to improve compatibility with the operative microscope with the METRx-MD system (Medtronic Sofamor Danek, Memphis, TN).

\section{CLINICAL MATERIAL AND METHODS}

The procedure involving placement of the METRx-MD system is similar to the MED that has been previously described in detail. ${ }^{5,8}$ Briefly, a paramedian muscle-splitting approach is facilitated by dilation of the paraspinous muscles with sequential dilators (Fig. 1). Visualization is then achieved using tubular retractors of various diameters and lengths. Special instruments, which have been optimized for use with the tubular retractors and the microscope, mirror those instruments commonly used for microdiscectomy. The major difference between METRx- 


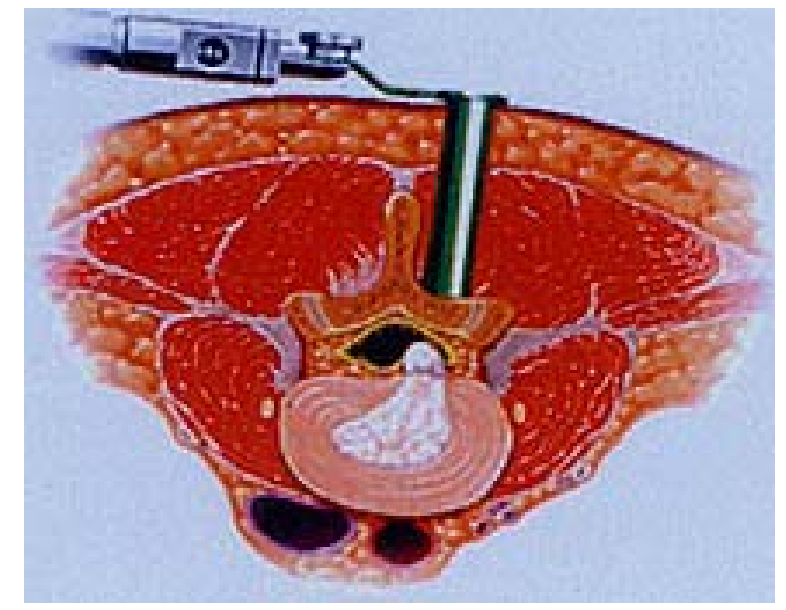

Fig. 1. Paramedian muscle splitting approach with tubular retractor in place. Figure reproduced with permission of Medtronic Sofamor Danek.

MD and MED is the use of the microscope, in the former, and the endoscope, in the latter, for intraoperative visualization.

Microendoscopic discectomy involving the METRxMD system was performed in 135 consecutive patients for lumbar herniated nucleus pulposi. During the study period, all elective surgeries performed by the author for virgin herniated nucleus pulposi were conducted using this method. All operations were planned as outpatient procedures involving the induction of general anesthesia. Preoperatively patients received dexamethasone $(20 \mathrm{mg})$ and antibiotic agents. The microscope was used with a prototype METRx instrument set, until the availability of the production set. All procedures were performed with patients in the prone position, positioned on a Wilson frame. At the conclusion of the procedure $80 \mathrm{mg}$ Depomedrol was injected epidurally at the operative site unless a durotomy had occurred. Bupivacaine $0.25 \%$ with 1:200,000 epinephrine $(10 \mathrm{ml})$ was infiltrated into the local tissues. The incision was closed in layers by using Vicryl (Johnson \& Johnson/Ethicon, Somerville, NJ) followed by Steristrips (3M Corporation, St. Paul, MN) and a bioocclusive dressing or, more recently, Dermabond (Johnson \& Johnson) on the skin edge. The patients were encouraged to use ice postoperatively and were given only oral analgesic medication (usually hydrocodone). They were observed in the outpatient center and discharged to home after they were ambulatory and had voided.

All patients completed a consent form, Patient Questionnaire-A form, and an SF-36 form prior to surgery. Office follow-up visits were conducted at 2 weeks, 6 weeks, and 3 months. Office follow up was extended when clinically indicated. Patients received questionnaires by mail from PhDx Systems, Inc. (Albuquerque, NM), at 6 weeks, 6 months, and 1 year postoperatively. Materials included a follow-up Patient Questionnaire-B form and an SF-36 form. Individuals at $\mathrm{PhDx}$ Systems tabulated the data, and the results were mailed to the operating surgeon quarterly. The data presented at this time are those obtained by the author at 1 year follow up and constitute his experience as part of an ongoing 12-site cooperative study.

\section{RESULTS}

One hundred thirty-five consecutive patients underwent discectomies involving placement of the METRX-MD system. Data were collected in $129(96 \%)$ of these patients. There were equal numbers of male and female patients, whose mean age was 42 years. Nonsmokers comprised $88 \%$ of the group. Workers' compensation was being received by $9 \%$, and $5 \%$ were involved in liability cases. Four patients suffered significant comorbid medical conditions. The mean length of symptoms prior to surgery was 25.5 weeks. Conservative care included pain management in $78 \%$, bedrest in $67 \%$, physical therapy in $51 \%$, epidural steroid therapy in $46 \%$, and chiropractic care in $32 \%$.

The disc herniation was contained in $56 \%$ and extruded in $44 \%$ of cases. Disc sizes ranged from 5 to $30 \mathrm{~mm}$ (Fig. 2 ). The most common location of the herniation was at the level of the intervertebral disc space; however, several large cephalad and caudally migrated fragments were removed. The caudally located fragments were often found in the axilla of the nerve root. The mean operative time was 66 minutes, and the mean blood loss was $22 \mathrm{ml}$. Intraoperative complications included three durotomies that were not primarily repaired but covered with gelfoam. These patients were not given epidural steroid agents and were admitted for 48 hours of bedrest. No delayed cerebrospinal fluid leaks or pseudomenigoceles developed. Excessive bleeding, arbitrarily defined as greater than 100 $\mathrm{ml}$, occurred in three patients.

Postoperative complications included one case of superficial wound infection, which resolved after local wound care and oral antibiotic therapy, and one case of discitis in which the patient presented with increasing back pain and required readmission; the latter resolved after a course of antibiotic therapy. Four patients required reoperation for recurrent disc herniations (the first underwent standard microdiscectomy and the last three underent surgery involving the METRx-MD system). Two of the reherniations occurred at the same level and on the same side, and two occurred at the same level but on the contralateral

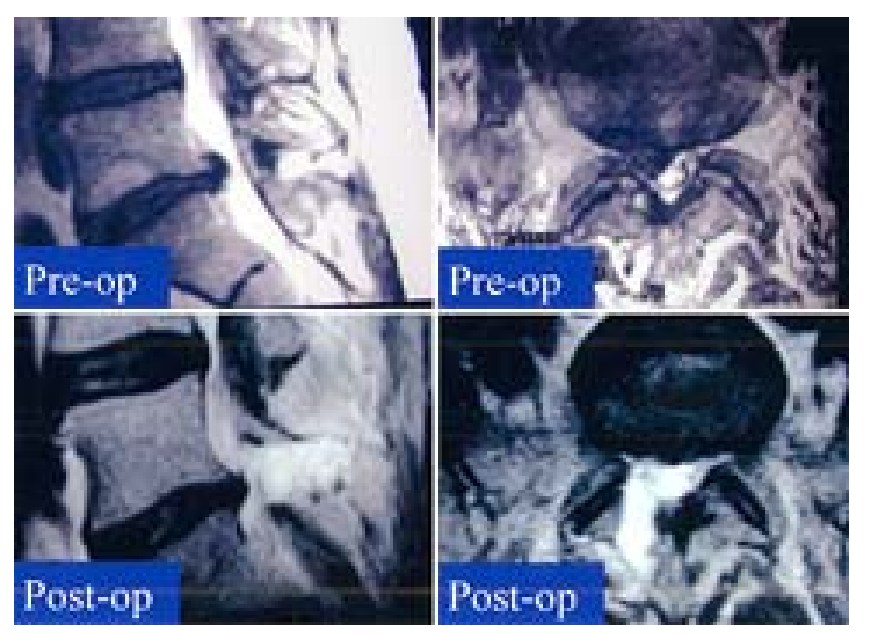

Fig. 2. Pre-and postoperative sagittal and axial magnetic resonance images of L5-S1 herniated nucleus pulposus. Figure reproduced with permission of Medtronic Sofamor Danek. 
side. One patient required reoperation for a contralateral spinal stenosis causing new contralateral pain. No neural injuries occurred.

The surgeon recorded data concerning the presence of back pain, radicular pain, motor deficit, reflex deficit, sensory deficit, and bowel/bladder dysfunction preoperatively and at the 2-, 6-, and 12-week office visits. Back pain was present in $99 \%$ of patients preoperatively and in $62 \%$ at 12 weeks postoperatively; radicular pain in 99 and $37 \%$, respectively; motor deficit in 65 and $16 \%$, respectively; reflex deficit in 48 and $33 \%$, respectively; and sensory deficit in 54 and 20\%, respectively. Preoperatively bowel/ bladder dysfunction was demonstrated in two patients, in both of whom, it resolved by the 2 -week visit. Back pain at the 12-week follow-up visit was improved in $97 \%$, worsened in $1 \%$, and unchanged in $2 \%$; radicular pain at 12 weeks was improved in $98 \%$, worsened in $1 \%$, and unchanged in 1\%; and sensory deficit at 12 weeks was improved in $99 \%$ and unchanged in $1 \%$.

The patients reported results to $\mathrm{PhDx}$ by mail surveys at 6 weeks, 6 months, and 1 year postoperatively. With regard to back pain, the mean pain discomfort scores measured on a VAS at $0,7,42,182$, and 365 days postoperatively were 6.2 (125 cases), 1.5 (46 cases), 2.1 (90 cases), 2.3 (84 cases), and 2.2 (72 cases), respectively. With regard to leg pain, the mean VAS pain scores were 7.3 (125 cases), 1.4 (51 cases), 1.9 (96 cases), 1.5 (84 cases), and 1.9 (73 cases), respectively. The ODI scores recorded during the same intervals for back pain were 53.8 (127 cases), 13.1 (51 cases), 19.3 (95 cases), 19.0 (87 cases), and 19.4 (73 cases), respectively; for leg pain they were 60.6 (124 cases), 12.8 (51 cases), 16 (95 cases), 13.4 (86 cases), and 13.3 (74 cases), respectively. The SF-36 physical health component subscale scores at $0,42,182$, and 365 days postoperatively were 28.2 (123 cases), 39.6 (96 cases), 45.6 (84 cases), and 45.4 (73 cases), respectively. The SF36 role limitations-physical subscale scores at the same intervals were 5.8 (125 cases), 32.2 (97 cases), 64.9 (87 cases), and 65.2 (74 cases), respectively. The SF-36 mental health component subscale scores at the same intervals were 46.2 (123 cases), 53 (96 cases), 54.0 (84 cases), and 53.2 (73 cases), respectively.

Patient satisfaction with physician, hospital, and office services, as reported to $\mathrm{PhDx}$, was scored on a scale from 0 to 100 (with 0 being poor to 100 being excellent). Satisfaction with services ranged from 93.4 to 76.9 (mean 88.3). Patient satisfaction with results at 2, 6, 26, and 52 weeks as reflected by answers to the question, ".... all things considered, they would have the surgery again for the same condition," was $96.2 \%$ (52 cases), $94.9 \%$ (97 cases), 93.4\% (87 cases), and 93.9\% (74 cases), respectively.

Return-to-work times were tabulated for those patients who were previously employed. The percentage of patients returning to work was $48.9 \%$ ( 22 cases) at 1 week, $78.8 \%$ (63 cases) at 6 weeks, 94.4\% (67 cases) at 6 months, and $93.1 \%$ (54 cases) at 1 year. Return-to-work time was tabulated only for those patients who supplied a valid return-to-work date. Mean return-to-work time was 32.1 days. The percentage of patients returning to work at various intervals was $8.9 \%$ in less than 1 week, $26.6 \%$ in 1 to 2 weeks, $38 \%$ in 3 to 5 weeks, $25.3 \%$ in 6 weeks to 6 months, and $1.3 \%$ in 6 months to 1 year.
Hospital charges averaged \$10,877 for METRx-MDtreated disc herniation procedures. Disc herniations treated by inpatient microdiscectomy, during the same time period, were associated with a mean hospital charge of $\$ 13,272$. The former represented a mean savings of $\$ 2395$ per case, or $18 \%$. The mean LOS for a METRx-MD-treated patient was 1 day ( 1 day was arbitrarily assigned for an outpatient procedure). The mean LOS for a microdiscectomy-treated patient was 2 days. No consideration was given to charges for admission of the three patients with dural tears or readmission for patients requiring reoperation.

\section{DISCUSSION}

Minimally invasive techniques in all areas of surgery have gained momentum in recent years. Spinal surgery has been no exception. Unfortunately, minimally invasive techniques have often been equated with minimally effective procedures. The procedure involving METRx-MD instrumentation and technique is an attempt to allow for a standard familiar microsurgical discectomy to be performed using standard microsurgical techniques via a minimally invasive approach. This is in keeping with the 1986 statement on the role of microdiscectomy in relation to standard discectomy in which Hudgins wrote, "My concept of micro-lumbar discectomy is that it consists of the ability to do all the surgical maneuvers of standard partial hemilaminectomy that have stood the test of time, but through a much smaller incision."10

It is important that the complication rate associated with the METRx-MD lumbar discectomy is comparable with that in standard microdiscectomy series. In our current series there was a $0.8 \%$ wound infection rate, a $0.80 \%$ discitis rate, and a $2.33 \%$ durotomy rate. These rates compared favorably with those reported by Williams ${ }^{23}(0,0$, and $0 \%$, respectively), Ebling, et al., ${ }^{6}(3.3,0.8$, and $3.9 \%$, respectively), Caspar, et al., ${ }^{3}(0.7,0.7$, and $6.7 \%$, respectively), and Pappas, et al., ${ }^{17}(7.2,0.5$, and $1 \%$, respectively). Our reoperation rate was $4 \%$. This included two reherniations at the same level and same side, two reherniations at the same level and contralateral side, and one reoperation for a contralateral spinal stenosis. The aforementioned authors reported reoperation rates of $14,5.5$, 5.7 , and $3 \%$, respectively.

The success rates are in general quite high with all surgical procedures for herniated lumbar discs. ${ }^{18}$ Williams, ${ }^{23}$ Ebling, et al., ${ }^{6}$ Caspar, et al., ${ }^{3}$ and Findlay, et al., ${ }^{7}$ have reported success rates ranging from 73 to $86 \%$. In our series $93.9 \%$ of patients answered affirmatively to the questions, "All things considered, I would have the surgery again." The VAS scores for low-back pain decreased from a mean of 6.2 prior to surgery to 2.21 year after surgery. The VAS scores for leg pain decreased from 7.3 to 1.9 over the same period. The SF-36 physical health component subscale score improved from 28.2 preoperatively to 45.41 year postoperatively. The mean SF-36 role limitation-physical score improved from 5.8 to 65.2 during the same interval.

Another measure of success is reflected by the patient's ability to return to previous employment. Bookwalter, et al., ${ }^{1}$ reported that $40 \%$ of their patients returned to work in 
fewer than 5 weeks. Caspar, et al., ${ }^{3}$ reported a mean return-to-work time of 18.6 weeks, and Foley and Smith ${ }^{8}$ reported a mean return-to-work time of 17.6 days. Our return-to-work time of 32 days is comparable with these series.

One of the driving forces behind minimally invasive surgery is economics. Less invasiveness often equates with shorter LOS, reduced morbidity, and quicker convalescence. This should result in greater cost effectiveness. Bookwalter, et al., ${ }^{1}$ reported a $91 \%$ success rate at performing standard microdiscectomies on an outpatient basis. They achieved a cost savings of $27 \%$. In 1994, New$\operatorname{man}^{15}$ reported on 74 patients who underwent outpatient microdiscectomies. He achieved a cost savings of $31 \%$. In comparing our series of outpatient METRx-MD-treated patients undergoing lumbar discectomies with a concurrent group treated as inpatients, the mean hospital charges decreased from $\$ 13,272$ to $\$ 10,877$, realizing a net savings of $\$ 2395$.

\section{CONCLUSIONS}

Minimally invasive surgery in which METRx-MD instrumentation is used is a clinically and cost-effective treatment for lumbar herniated discs. Results and complications were comparable with those associated with standard microdiscectomy techniques. Patient satisfaction was high (93.9\%), and a mean cost savings of \$2395 was realized.

\section{Disclosure}

Sylvain Palmer, M.D., acts as a consultant for Medtronic Sofamor Danek, which provided technical and financial support.

\section{References}

1. Bookwalter JW III, Busch MD, Nicely D: Ambulatory surgery is safe and effective in radicular disc disease. Spine 19: 536-530, 1994

2. Caspar W: A new surgical procedure for lumbar disk herniation causing less tissue damage through a microsurgical approach. Adv Neurosurg 4:74-80, 1977

3. Caspar W, Campbell B, Barbier D, et al: The Caspar microsurgical discectomy and comparison with a conventional standard lumbar disc procedure. Neurosurgery 28:78-87, 1991

4. Davis H: Increasing rates of cervical and lumbar spine surgery in the United States, 1979-1990. Spine 19:1117-1124, 1994

5. Dirksmeier PJ, Parsons IM IV, Kang JD: Microendoscopic and open laminotomy and discectomy in lumbar disc disease. Semin Spine Surg 11:138-146, 1999

6. Ebeling U, Reichenberg W, Reulen HJ: Results of microsurgi- cal lumbar discectomy. Review on 485 patients. Acta Neurochir 81:45-52, 1986

7. Findlay GF, Hall BI, Musa BS, et al: A 10-year follow-up of the outcome of lumbar microdiscectomy. Spine 23:1168-1171, 1998

8. Foley KT, Smith MM: Microendoscopic discectomy. Techn Neurosurg 3:301-307, 1997

9. Holbrook TL, Grozier K, Kelsey JL, et al: The Frequency of Occurrence, Impact, and Cost of Selected Musculoskeletal Conditions in the United States. Chicago: American Academy of Orthopedic Surgeons, 1984, pp 154-156

10. Hudgins WR: The role of microdiscectomy. Orthop Clin North Am 14:589-603, 1983

11. Kambin P, O'Brien E, Zhou L, et al: Arthroscopic microdiscectomy and selective fragmentectomy. Clin Orthop 347: 150-167, 1998

12. Mixter WJ, Barr JS: Rupture of the intervertebral disc with involvement of the spinal canal. N Engl J Med 211:210-215, 1934

13. Nachemson A: The lumbar spine: an orthopedic challenge. Spine 1:59-71, 1976

14. Nachemson A: Advances in low-back pain. Clin Orthop Relat Res 200:266-278, 1985

15. Newman MH: Outpatient conventional laminotomy and disc excision. Spine 20:353-355, 1995

16. Onik G, Mooney V, Maroon JC, et al: Automated percutaneous discectomy: a prospective multi-institutional study. Neurosurgery 26:228-233, 1990

17. Pappas CT, Harrington T, Sonntag VK: Outcome analysis in 654 surgical treated lumbar disc herniations. Neurosurgery 30: 862-866, 1992

18. Tullberg T, Isacson J, Weidenhielm L: Does microscopic removal of lumbar disc herniation lead to better results than the standard procedure? Results of a one-year randomized study. Spine 18:24-27, 1993

19. Saal JA, Saal JS, Herzog RJ: The natural history of lumbar intervertebral disc extrusions treated nonoperatively. Spine 15: 683-686, 1990

20. Smith L: Enzyme dissolution of the nucleus pulposus in humans. JAMA 187:137-140, 1964

21. van Tulder MW, Koes BW, Bouter LM: Conservative treatment of acute and chronic nonspecific low back pain. A systematic review of randomized controlled trials of the most common intervention. Spine 22:2128-2156, 1997

22. Weber H: Lumbar disc herniation. A controlled, prospective study with ten years of observation. Spine 8:131-140, 1983

23. Williams RW: Microlumbar discectomy: a conservative surgical approach to the virgin herniated lumbar disc. Spine 3: $175-182,1978$

Manuscript received June 17, 2002.

Accepted in final form July 3, 2002.

Address reprint requests to: Sylvain Palmer, M.D., 26732 Crown Valley Parkway, Suite 561, Mission Viejo, California 92651. email: sylvainpalmer@cox.net. 